\title{
Perturbation theorems for local integrated semigroups
}

\author{
by \\ Chung-Cheng Kuo (Taipei)
}

\begin{abstract}
We apply the contraction mapping theorem to establish some bounded and unbounded perturbation theorems concerning nondegenerate local $\alpha$-times integrated semigroups. Some unbounded perturbation results of Wang et al. [Studia Math. 170 (2005)] are also generalized. We also establish some growth properties of perturbations of local $\alpha$-times integrated semigroups.
\end{abstract}

1. Introduction. Let $X$ be a Banach space with a norm $\|\cdot\|$, and $L(X)$ the set of all bounded linear operators on $X$. For each $\alpha>0$ and $0<T_{0} \leq \infty$, a family $S(\cdot)\left(=\left\{S(t) \mid 0 \leq t<T_{0}\right\}\right)$ in $L(X)$ is called a local $\alpha$-times integrated semigroup on $X$ if it is strongly continuous and satisfies

$$
S(t) S(s) x=\frac{1}{\Gamma(\alpha)}\left[\int_{0}^{t+s}-\int_{0}^{t}-\int_{0}^{s}\right](t+s-r)^{\alpha-1} S(r) x d r
$$

for all $x \in X$ and $0 \leq t, s \leq t+s<T_{0}$ (see [12, 14, 16]). Here $\Gamma(\cdot)$ denotes the Gamma function. Moreover, we say that $S(\cdot)$ is

(1.2) locally Lipschitz continuous if for each $0<t_{0}<T_{0}$ there exists a $K_{t_{0}}>0$ such that $\|S(t+h)-S(t)\| \leq K_{t_{0}} h$ for all $0 \leq t, h \leq t+h \leq t_{0}$;

(1.3) exponentially bounded if there exist $K, \omega \geq 0$ such that $\|S(t)\| \leq K e^{\omega t}$ for all $t \geq 0$;

(1.4) exponentially Lipschitz continuous if there exist $K, \omega \geq 0$ such that $\|S(t+h)-S(t)\| \leq K h e^{\omega(t+h)}$ for all $t, h \geq 0$

(1.5) nondegenerate if $x=0$ whenever $S(t) x=0$ for all $0 \leq t<T_{0}$. In this case, the (integral) generator of $S(\cdot)$ is defined by $D(A)=$ $\left\{x \in X \mid y_{x} \in X\right.$ and $S(t) x-j_{\alpha}(t) x=\int_{0}^{t} S(r) y_{x} d r$ for all $\left.0 \leq t<T_{0}\right\}$ and $A x=y_{x}$ for each $x \in D(A)$. Here $j_{\beta}(t)=t^{\beta} / \Gamma(\beta+1)$ for $\beta>-1$ and $t>0$.

A local $\alpha$-times integrated semigroup is called an $\alpha$-times integrated semigroup if $T_{0}=\infty$ (see [1 9, 14, 26, 27]). In general, an $\alpha$-times inte-

Key words and phrases: integrated semigroup, generator, abstract Cauchy problem. 
grated semigroup may not be exponentially bounded and the generator of a nondegenerate local $\alpha$-times integrated semigroup may not be densely defined.

The problem of bounded perturbations of (local) $\alpha$-times integrated semigroups has been extensively studied by many authors [1, 4, 5, 11, 14, 15, [21, 25 27]. In particular, Xiao and Liang [25, Theorem 1.3.5] show that if $A$ generates an exponentially bounded nondegenerate $\alpha$-times integrated semigroup on $X$ and $B$ is a bounded linear operator on $X$ such that $B A \subset A B$, then $A+B$ generates an exponentially bounded nondegenerate $\alpha$-times integrated semigroup on $X$; this has been extended by the author in [11] to the case when $B$ is only a bounded linear operator on $\overline{D(A)}$, and $\mathrm{Li}$ and Shaw [15] show that if $B$ is a bounded linear operator on $X$ which commutes with $S(\cdot)$ on $X$, then $A+B$ generates a nondegenerate $\alpha$-times integrated semigroup on $X$ which may not be exponentially bounded; this result is also extended to the context of local $\alpha$-times integrated semigroups in [13] by another method. Recently, some unbounded perturbation theorems concerning local $\alpha$-times integrated semigroups are also established in [15, 25] and some interesting applications of this topic are illustrated in [1-8, 25, 26]. In particular, Wang et al. [25] show that $A+B$ generates a local $\alpha$-times integrated semigroup if $\alpha \in \mathbb{N}$ and $B$ is a bounded linear operator on $[D(A)]$ such that $B x \in D\left(A^{l+1}\right)$ for all $x \in D(A)$ and either $A+B$ is a closed linear operator or $A B=B A$ on $D\left(A^{2}\right)$. Here $l$ denotes the smallest nonnegative integer that is larger than or equal to $\alpha$.

The purpose of this paper is to investigate several bounded and unbounded additive perturbation theorems for local $\alpha$-times integrated semigroups on $X$. Growth properties of perturbations are also established. In Section 2, we show that if $A$ generates a nondegenerate local $\alpha$-times integrated semigroup $S(\cdot)$ on $X$ and if $B$ is a bounded linear operator from $\overline{D(A)}$ into $X$ such that $B x \in D\left(A^{l}\right)$ for all $x \in \overline{D(A)}$, then $A+B$ generates a nondegenerate local $\alpha$-times integrated semigroup $T(\cdot)$ on $X$ satisfying $T(\cdot) x=S(\cdot) x+D^{\alpha} S * B T(\cdot) x$ on $\left[0, T_{0}\right)$ for all $x \in X$ (Theorem 2.10); this has been obtained by Nicaise in [21, Corollary 4.2] using a Hille-Yosida space argument (see [4, 5]) when $\alpha \in \mathbb{N}$ and $T_{0}=\infty$. Moreover, $T(\cdot)$ is exponentially bounded (resp., norm continuous or exponentially Lipschitz continuous) if $S(\cdot)$ is. We then show that $T(\cdot)$ is also locally Lipschitz continuous if $S(\cdot)$ is and $B x \in D\left(A^{l-1}\right)$ for all $x \in \overline{D(A)}$ (Theorem 2.12); this has been obtained by Kellermann and Hieber in [9] when $\alpha=1$.

In Section 3, we first show that if $B$ is a bounded linear operator from $[D(A)]$ into $X$ such that $B x \in D\left(A^{l+1}\right)$ for all $x \in D(A)$ and $A+B$ is a closed linear operator from $D(A)$ into $X$, then $A+B$ generates a nondegenerate local $\alpha$-times integrated semigroup $T(\cdot)$ on $X$ satisfying $T(\cdot) x=$ $S(\cdot) x+D^{\alpha+1} S * B \widetilde{T}(\cdot) x$ on $\left[0, T_{0}\right)$ for all $x \in X($ Theorem 3.1$)$. Moreover, $T(\cdot)$ 
is exponentially bounded (resp., norm continuous or exponentially Lipschitz continuous) if $S(\cdot)$ is. We then show that $T(\cdot)$ is also locally Lipschitz continuous if $S(\cdot)$ is and $B x \in D\left(A^{l}\right)$ for all $x \in D(A)$ (Theorem 3.2). Here $\widetilde{T}(\cdot)=$ $j_{0} * T(\cdot)$. We also show that the nondegenerate local $\alpha$-times integrated semigroup $T(\cdot)$ on $X$ satisfies $T(\cdot) x=S(\cdot) x+D^{\alpha} S *(\lambda-A) B(\lambda-A)^{-1} T(\cdot) x$ on $\left[0, T_{0}\right)$ for all $x \in X$ if the assumption $A B=B A$ on $D\left(A^{2}\right)$ is added (Corollaries 3.5 and 3.6). Here $\lambda \in \rho(A)$ (the resolvent set of $A$ ) is fixed. An illustrative example concerning these theorems is also presented in the final part of this paper.

2. Bounded perturbation theorems. In this section, we first recall some basic properties of a nondegenerate local $\alpha$-times integrated semigroup and known results about connections between the generator of such a semigroup and strong solutions of the abstract Cauchy problem

$$
A C P(A, f, x)\left\{\begin{array}{l}
u^{\prime}(t)=A u(t)+f(t) \quad \text { for } t \in\left(0, T_{0}\right), \\
u(0)=x,
\end{array}\right.
$$

where $x \in X$ and $f$ is an $X$-valued function defined on $\left(0, T_{0}\right)$.

Proposition 2.1 (see [10, 14, 16, 18). Let $A$ be the generator of a nondegenerate local $\alpha$-times integrated semigroup $S(\cdot)$ on $X$. Then

(2.1) $\quad S(0)=0$ (the zero operator) on $X$;

(2.2) $\quad A$ is closed and $\rho(A)$ (the resolvent set of $A$ ) is nonempty;

(2.3) $S(t) x \in D(A)$ and $S(t) A x=A S(t) x$ for $x \in D(A)$ and $0 \leq t<T_{0}$;

(2.4) $\quad \int_{0}^{t} S(r) x d r \in D(A)$ and $A \int_{0}^{t} S(r) x d r=S(t) x-j_{\alpha}(t) x$ for $x \in X$ and $0 \leq t<T_{0}$;

(2.5) $\quad R(S(t)) \subset \overline{D(A)}$ for $0 \leq t<T_{0}$;

(2.6) for each $\beta>\alpha, j_{\beta-\alpha-1} * S(\cdot)$ is a nondegenerate local $\beta$-times integrated semigroup on $X$ with generator $A$.

Definition 2.2. Let $A: D(A) \subset X \rightarrow X$ be a closed linear operator in a Banach space $X$ with domain $D(A)$ and range $R(A)$. A function $u:\left[0, T_{0}\right) \rightarrow X$ is called a (strong) solution of $A C P(A, f, x)$ if $u \in$ $C^{1}\left(\left(0, T_{0}\right), X\right) \cap C\left(\left[0, T_{0}\right), X\right) \cap C\left(\left(0, T_{0}\right),[D(A)]\right)$ and satisfies $A C P(A, f, x)$. Here $[D(A)]$ denotes the Banach space $D(A)$ with the norm $|\cdot|$ defined by $|x|=\|x\|+\|A x\|$ for all $x \in D(A)$.

Remark 2.3. $u \in C\left(\left[0, T_{0}\right),[D(A)]\right)$ if $f \in C\left(\left[0, T_{0}\right), X\right)$ and $u$ is a (strong) solution of $A C P(A, f, x)$ in $C^{1}\left(\left[0, T_{0}\right), X\right)$.

Theorem 2.4 (see [12]). A generates a nondegenerate local $\alpha$-times integrated semigroup $S(\cdot)$ on $X$ if and only if for each $x \in X, A C P\left(A, j_{\alpha}(\cdot) x, 0\right)$ has a unique (strong) solution $u(\cdot, x)$ in $C^{1}\left(\left[0, T_{0}\right), X\right)$. In this case, we have $u(\cdot, x)=j_{0} * S(\cdot) x$ for all $x \in X$. 
We next recall some results concerning the $\alpha$ th derivative of a continuous function from a subinterval $I$ of $\left[0, T_{0}\right)$ containing $\{0\}$ into $X$ which have been given in [12].

Definition 2.5. Let $\alpha>0, k=[\alpha]+1$ and $v: I \rightarrow X$ for some subinterval $I$ of $\left[0, T_{0}\right)$ containing $\{0\}$. We write $v \in C^{\alpha}(I, X)$ if $v=v(0)+$ $j_{\alpha-k} * u$ on $I$ for some $u \in C^{k-1}(I, X)$. In this case, we say that $v$ is $\alpha$-times continuously differentiable on $I$, and the $(k-1)$ th derivative of $u$ on $I$ is called the $\alpha$ th derivative of $v$ on $I$ and denoted by $D^{\alpha} v$ (on $I$ ) or $D^{\alpha} v: I \rightarrow X$. Here $C^{k}(I, X)$ denotes the set of all $k$-times continuously differentiable functions from $I$ into $X$, and $C^{0}(I, X)=C(I, X)$ the set of all continuous functions from $I$ into $X$.

REMARK 2.6 (see [10]). Let $k=[\alpha]+1$ and $v \in C^{\alpha}(I, X)$ for some subinterval $I$ of $\left[0, T_{0}\right)$ containing $\{0\}$. Assume that $v(0)=0$. Then $j_{k-\alpha-1} *$ $v \in C^{k}(I, X), v \in C^{\alpha-i}(I, X)$ and $D^{\alpha-i} v=\left(j_{k-\alpha-1} * v\right)^{(k-i)}$ on $I$ for all integers $0 \leq i \leq k-1$. In particular, $j_{\alpha}(\cdot) \in C^{\alpha}\left(\left[0, T_{0}\right), \mathbb{C}\right)$ and $D^{\alpha-i} j_{\alpha}(\cdot)=$ $D^{k-i} j_{k}(\cdot)=j_{i}(\cdot)$ on $\left[0, T_{0}\right)$ for all integers $0 \leq i \leq k-1$.

Proposition 2.7 (see [10]). Let $A$ be the generator of a nondegenerate local $\alpha$-times integrated semigroup $S(\cdot)$ on $X, x \in X$ and $f \in L_{\text {loc }}^{1}\left(\left[0, T_{0}\right), X\right) \cap$ $C\left(\left(0, T_{0}\right), X\right)$. Then $A C P(A, f, x)$ has a (strong) solution u in $C^{1}\left(\left[0, T_{0}\right), X\right)$ if and only if $v(\cdot)=S(\cdot) x+S * f(\cdot) \in C^{\alpha+1}\left(\left[0, T_{0}\right), X\right)$. In this case, $u=D^{\alpha} v$ on $\left[0, T_{0}\right)$.

Lemma 2.8 (see [10]). Let $V(\cdot)$ and $Z(\cdot)$ be strongly continuous families of bounded linear operators from $X$ into some Banach space $Y$, and let $W(\cdot)$ be a strongly continuous family in $L(Y)$ such that $Z(\cdot) x=V(\cdot) x+W * Z(\cdot) x$ on $\left[0, T_{0}\right)$ for all $x \in X$. Then $Z(\cdot)$ is exponentially bounded (resp., norm continuous or exponentially Lipschitz continuous) if $V(\cdot)$ and $W(\cdot)$ both are.

By slightly modifying the proof of [22, Lemma 2.11] we can obtain the next lemma.

Lemma 2.9. Let $V(\cdot)$ be a locally Lipschitz continuous family of bounded linear operators from $X$ into some Banach space $Y$, and let $W(\cdot)$ be a locally Lipschitz continuous family in $L(Y)$ with $W(0)=0$ on $Y$. Then there exists a unique locally Lipschitz continuous family $Z(\cdot)$ of bounded linear operators from $X$ into $Y$ such that

$$
Z(t) x=V(t) x+\frac{d}{d t} W * Z(t) x
$$

for all $x \in X$ and $t \in\left[0, T_{0}\right)$.

The next theorem is a bounded perturbation of local $\alpha$-times integrated semigroups on $X$ which has been established by Nicaise in [21, Corollary 4.2] using a Hille-Yosida space argument when $\alpha \in \mathbb{N}$ and $T_{0}=\infty$. 
TheOREM 2.10. Let $S(\cdot)$ be a nondegenerate local $\alpha$-times integrated semigroup on $X$ with generator $A$. Assume that $B$ is a bounded linear operator from $\overline{D(A)}$ into $X$ such that $B x \in D\left(A^{l}\right)$ for all $x \in \overline{D(A)}$. Then $A+B$ generates a nondegenerate local $\alpha$-times integrated semigroup $T(\cdot)$ on $X$ satisfying

$$
T(\cdot) x=S(\cdot) x+D^{\alpha} S * B T(\cdot) x \quad \text { on }\left[0, T_{0}\right)
$$

for all $x \in X$. Moreover, $T(\cdot)$ is also exponentially bounded (resp., norm continuous or exponentially Lipschitz continuous) if $S(\cdot)$ is.

Proof. Indeed, if we set $k=[\alpha]+1$, we may define $\widetilde{S}(t): X \rightarrow X$ for $0 \leq t<T_{0}$ by $\widetilde{S}(t) x=j_{k-\alpha-1} * S(t) x$ for all $x \in X$. By $(2.6), \widetilde{S}(\cdot)$ is a nondegenerate local $k$-times integrated semigroup on $X$ with generator $A$. It is also easy to see from (2.3) and (2.4) that

$$
\widetilde{S}(t) y=j_{r-1} * \widetilde{S}(t) A^{r} y+\sum_{i=0}^{r-1} j_{k+i}(t) A^{i} y
$$

for all $r \in \mathbb{N}, y \in D\left(A^{r}\right)$ and $0 \leq t<T_{0}$. Combining (2.8) with Remark 2.6, we have

$$
\begin{aligned}
& D^{\alpha}(S * B f)(\cdot)=D^{k}(\widetilde{S} * B f)(\cdot) \\
& = \begin{cases}D^{k}\left(j_{k-2} * \widetilde{S} * A^{k-1} B f+\sum_{i=0}^{k-2} j_{k+i} * A^{i} B f\right)(\cdot) & \text { if } \alpha=k-1 \in \mathbb{N} \\
D^{k}\left(j_{k-1} * \widetilde{S} * A^{k} B f+\sum_{i=0}^{k-1} j_{k+i} * A^{i} B f\right)(\cdot) & \text { if } k-1<\alpha<k\end{cases} \\
& = \begin{cases}S * A^{k-1} B f(\cdot)+\sum_{i=0}^{k-2} j_{i} * A^{i} B f(\cdot) & \text { if } \alpha=k-1 \in \mathbb{N} \\
\widetilde{S} * A^{k} B f(\cdot)+\sum_{i=0}^{k-1} j_{i} * A^{i} B f(\cdot) & \text { if } k-1<\alpha<k\end{cases}
\end{aligned}
$$

on $\left[0, t_{0}\right]$ for all $0<t_{0}<T_{0}$ and $f \in C\left(\left[0, t_{0}\right], \overline{D(A)}\right)$. We shall show that for each $x \in X$ there exists a unique function $w_{x}$ in $C\left(\left[0, T_{0}\right), \overline{D(A)}\right)$ such that $w_{x}(\cdot)=S(\cdot) x+D^{\alpha} S * B w_{x}(\cdot)$ on $\left[0, T_{0}\right)$; this may be done by using Theorem 2.4. Indeed, fix $x \in X$ and $0<t_{0}<T_{0}$ and define $U: C\left(\left[0, t_{0}\right], \overline{D(A)}\right) \rightarrow C\left(\left[0, t_{0}\right], \overline{D(A)}\right)$ by $U(f)(\cdot)=S(\cdot) x+D^{\alpha}(S * B f)(\cdot)$ on $\left[0, t_{0}\right]$ for all $f \in C\left(\left[0, t_{0}\right], \overline{D(A)}\right)$. From $(2.1),(2.5)$ and the assumption $B x \in D\left(A^{l}\right)$ for all $x \in \overline{D(A)}$, we see that $U$ is well-defined and $A^{i} B$ is a bounded linear operator from $\overline{D(A)}$ into $X$ for all integers $0 \leq i \leq l$. We first claim that 


$$
\left\|D^{\alpha} S * B f(t)\right\| \leq M_{t_{0}} \int_{0}^{t}\|f(s)\| d s
$$

for all $f \in C\left(\left[0, t_{0}\right], \overline{D(A)}\right)$ and $0 \leq t \leq t_{0}$, where

$$
M_{t_{0}}= \begin{cases}\sup _{0 \leq r \leq t_{0}}\|S(r)\|\left\|A^{k-1} B\right\|+\sum_{i=0}^{k-2} j_{i}\left(t_{0}\right)\left\|A^{i} B\right\| & \text { if } \alpha=k-1 \in \mathbb{N}, \\ \sup _{0 \leq r \leq t_{0}}\|\widetilde{S}(r)\|\left\|A^{k} B\right\|+\sum_{i=0}^{k-1} j_{i}\left(t_{0}\right)\left\|A^{i} B\right\| & \text { if } k-1<\alpha<k .\end{cases}
$$

To see this, we consider only the case $\alpha=k-1 \in \mathbb{N}$, for the case $k-1<\alpha<k$ can be treated similarly. Indeed, if $\alpha=k-1 \in \mathbb{N}$ and $f \in C\left(\left[0, t_{0}\right], \overline{D(A)}\right)$, then

$$
\begin{aligned}
\left\|S * A^{k-1} B f(t)\right\| & \leq \int_{0}^{t}\left\|S(t-s) A^{k-1} B f(s)\right\| d s \\
& \leq \int_{0}^{t} \sup _{0 \leq r \leq t_{0}}\|S(r)\|\left\|A^{k-1} B\right\|\|f(s)\| d s \\
& =\sup _{0 \leq r \leq t_{0}}\|S(r)\|\left\|A^{k-1} B\right\| \int_{0}^{t}\|f(s)\| d s
\end{aligned}
$$

and

$$
\begin{aligned}
\left\|j_{i} * A^{i} B f(t)\right\| & \leq \int_{0}^{t}\left\|j_{i}(t-s) A^{i} B f(s)\right\| d s \\
& \leq \int_{0}^{t} j_{i}\left(t_{0}\right)\left\|A^{i} B\right\|\|f(s)\| d s \\
& =j_{i}\left(t_{0}\right)\left\|A^{i} B\right\| \int_{0}^{t}\|f(s)\| d s
\end{aligned}
$$

for all $0 \leq t \leq t_{0}$ and integers $0 \leq i \leq k-2$, and so

$$
\begin{aligned}
\left\|D^{\alpha} S * B f(t)\right\| & \leq\left\|S * A^{k-1} B f(t)\right\|+\sum_{i=0}^{k-2}\left\|j_{i} * A^{i} B f(t)\right\| \\
& \leq M_{t_{0}} \int_{0}^{t}\|f(s)\| d s
\end{aligned}
$$

for all $0 \leq t \leq t_{0}$. Hence (2.10) holds when $\alpha=k-1 \in \mathbb{N}$. By induction, we have 


$$
\begin{aligned}
\left\|U^{n} f(t)-U^{n} g(t)\right\| & =\left\|U\left(U^{n-1} f\right)(t)-U\left(U^{n-1} g\right)(t)\right\| \\
& =\left\|D^{\alpha} S * B\left(U^{n-1} f-U^{n-1} g\right)(t)\right\| \\
& \leq M_{t_{0}}^{n} \int_{0}^{t} j_{n-1}(t-s)\|f(s)-g(s)\| d s \\
& \leq M_{t_{0}}^{n} j_{n}(t)\|f-g\| \leq M_{t_{0}}^{n} j_{n}\left(t_{0}\right)\|f-g\|
\end{aligned}
$$

for all $f, g \in C\left(\left[0, t_{0}\right], \overline{D(A)}\right), 0 \leq t \leq t_{0}$ and $n \in \mathbb{N}$, where $\|f-g\|=$ $\max _{0 \leq s \leq t_{0}}\|f(s)-g(s)\|$. It follows from the contraction mapping theorem that there exists a unique function $w_{x, t_{0}}$ in $C\left(\left[0, t_{0}\right], \overline{D(A)}\right)$ such that $w_{x, t_{0}}(\cdot)=S(\cdot) x+D^{\alpha} S * B w_{x, t_{0}}(\cdot)$ on $\left[0, t_{0}\right]$. In this case, we set $w_{x}(t)=$ $w_{x, t_{0}}(t)$ for all $0 \leq t \leq t_{0}<T_{0}$. Then $w_{x}(\cdot)$ is a unique function in $C\left(\left[0, T_{0}\right), \overline{D(A)}\right)$ such that $w_{x}(\cdot)=S(\cdot) x+D^{\alpha} S * B w_{x}(\cdot)$ on $\left[0, T_{0}\right)$. Since $S * j_{\alpha}(\cdot) x+S * j_{0} * B w_{x} \in C^{\alpha+1}\left(\left[0, T_{0}\right), X\right)$ and $D^{\alpha}\left(S * j_{\alpha}(\cdot) x+S * j_{0} * B w_{x}\right)=$ $j_{0} * S(\cdot) x+j_{0} * D^{\alpha} S * B w_{x}=j_{0} * w_{x}$ on $\left[0, T_{0}\right)$, we deduce from Proposition 2.7 that $u=j_{0} * w_{x}$ is the unique (strong) solution of $A C P\left(A, j_{\alpha}(\cdot) x+j_{0} * B w_{x}, 0\right)$ in $C^{1}\left(\left[0, T_{0}\right), X\right)$, and so $u=j_{0} * w_{x}$ is the unique function in $C^{1}\left(\left[0, T_{0}\right), X\right)$ such that $u^{\prime}\left(=A u+j_{\alpha} x+j_{0} * B w_{x}=A u+j_{\alpha} x+B u\right)=(A+B) u+j_{\alpha} x$ on $\left[0, T_{0}\right)$. Hence $u=j_{0} * w_{x}$ is the unique (strong) solution of $A C P(A+$ $\left.B, j_{\alpha}(\cdot) x, 0\right)$ in $C^{1}\left(\left[0, T_{0}\right), X\right)$, which together with Theorem 2.4 implies that $A+B$ generates a nondegenerate $\alpha$-times integrated semigroup $T(\cdot)$ on $X$ satisfying (2.7). Combining Lemma 2.8 with $(2.9)$, we find that $T(\cdot)$ is also exponentially bounded (resp., norm continuous or exponentially Lipschitz continuous) if $S(\cdot)$ is, by setting $Y=\overline{D(A)}, V(\cdot)=S(\cdot), Z(\cdot)=T(\cdot)$ and

$$
W(\cdot)= \begin{cases}S(\cdot) A^{k-1} B+\sum_{i=0}^{k-2} j_{i}(\cdot) A^{i} B & \text { if } \alpha=k-1 \in \mathbb{N}, \\ \widetilde{S}(\cdot) A^{k} B+\sum_{i=0}^{k-1} j_{i}(\cdot) A^{i} B & \text { if } k-1<\alpha<k,\end{cases}
$$

in Lemma 2.8.

REMARK 2.11. Let $W(\cdot)$ be a locally Lipschitz continuous family in $L(Y)$ with $W(0)=0$ for some Banach space $Y$ and $g \in L_{\text {loc }}^{1}\left(\left[0, T_{0}\right), Y\right)$. Then $W * g \in C^{1}\left(\left[0, T_{0}\right), Y\right)$ and for each $0<t_{0}<T_{0}$, we have $\left\|(W * g)^{\prime}(t)\right\| \leq$ $K_{t_{0}} \int_{0}^{t}\|g(s)\| d s$ for all $0 \leq t \leq t_{0}$. Here $K_{t_{0}}$ is given as in (1.3) with $S(\cdot)$ is replaced by $W(\cdot)$. Moreover, $(W * g)^{\prime}(\cdot)$ is locally Lipschitz continuous if $g$ is.

By slightly modifying the proof of Theorem 2.10, we can establish the next bounded perturbation theorem concerning locally Lipschitz continuous local $\alpha$-times integrated semigroups on $X$, which has been obtained by Kellermann and Hieber in [9] when $\alpha=1$.

THEOREM 2.12. Let $A$ be the generator of a locally Lipschitz continuous nondegenerate local $\alpha$-times integrated semigroup $S(\cdot)$ on $X$ for some 
$\alpha \geq 1$. Assume that $B$ is a bounded linear operator from $\overline{D(A)}$ into $X$. Then $A+B$ generates a locally Lipschitz continuous nondegenerate local $\alpha$-times integrated semigroup $T(\cdot)$ on $X$ satisfying $(2.7)$, if either $\alpha=1$ or $\alpha>1$ with $B x \in D\left(A^{l-1}\right)$ for all $x \in \overline{D(A)}$.

Proof. Just as in the proof of Theorem 2.10, we shall first show that $A+B$ generates a nondegenerate local $\alpha$-times integrated semigroup $T(\cdot)$ on $X$ satisfying (2.7), and need only show that

$$
\left\|D^{\alpha} S * B f(t)\right\| \leq N_{t_{0}} \int_{0}^{t}\|f(s)\| d s
$$

for all $0<t_{0}<T_{0}, f \in C\left(\left[0, t_{0}\right], \overline{D(A)}\right)$ and $0 \leq t \leq t_{0}$. Here

$$
N_{t_{0}}= \begin{cases}K_{t_{0}}\|B\| & \text { if } \alpha=1, \\ K_{t_{0}} j_{k-\alpha}\left(t_{0}\right)\left\|A^{k-1} B\right\|+\sum_{i=0}^{k-2} j_{i}\left(t_{0}\right)\left\|A^{i} B\right\| & \text { if } 1 \leq k-1<\alpha<k, \\ K_{t_{0}}\left\|A^{k-2} B\right\|+\sum_{i=0}^{k-3} j_{i}\left(t_{0}\right)\left\|A^{i} B\right\| & \text { if } \alpha=k-1 \geq 2,\end{cases}
$$

and $K_{t_{0}}$ is given as in (1.3). Indeed, the local Lipschitz continuity of $S(\cdot)$ implies that $\widetilde{S}(\cdot)$ is also locally Lipschitz continuous with a Lipschitz constant $K_{t_{0}} j_{k-\alpha}\left(t_{0}\right)$ on $\left[0, t_{0}\right]$ for all $0<t_{0}<T_{0}$. Combining Remarks 2.6 and $2.11,(2.8)$ with the assumption $B x \in D\left(A^{l-1}\right)$ for all $x \in \overline{D(A)}$, we have $S * B f \in C^{\alpha}\left(\left[0, t_{0}\right], \overline{D(A)}\right)$ and

$$
= \begin{cases}(S * B f)^{\prime}(\cdot) & \text { if } \alpha=1, \\ D^{k}\left(j_{k-2} * \widetilde{S} * A^{k-1} B f+\sum_{i=0}^{k-2} j_{k+i} * A^{i} B f\right)(\cdot) & \\ =\left(\widetilde{S} * A^{k-1} B f\right)^{\prime}(\cdot)+\sum_{i=0}^{k-2} j_{i} * A^{i} B f(\cdot) & \text { if } 1 \leq k-1<\alpha<k, \\ D^{k}\left(j_{k-3} * \widetilde{S} * A^{k-2} B f+\sum_{i=0}^{k-3} j_{k+i} * A^{i} B f\right)(\cdot) & \\ =\left(S * A^{k-2} B f\right)^{\prime}(\cdot)+\sum_{i=0}^{k-3} j_{i} * A^{i} B f(\cdot) & \text { if } \alpha=k-1 \geq 2,\end{cases}
$$

on $\left[0, t_{0}\right]$ for all $0<t_{0}<T_{0}$ and $f \in C\left(\left[0, t_{0}\right], \overline{D(A)}\right)$. Now if $0<t_{0}<T_{0}$ is fixed, then for each $f \in C\left(\left[0, t_{0}\right], \overline{D(A)}\right)$ and $0 \leq t \leq t_{0}$, from Remark 2.11 and the continuity of $A^{i} B$ on $\overline{D(A)}$ for integers $1 \leq i \leq k-1$ we obtain 


$$
\left\|\left(S * A^{k-2} B f\right)^{\prime}(t)\right\| \leq K_{t_{0}} \int_{0}^{t}\left\|A^{k-2} B f(s)\right\| d s \leq K_{t_{0}}\left\|A^{k-2} B\right\| \int_{0}^{t}\|f(s)\| d s
$$

if $\alpha=k-1 \geq 1$, and

$$
\begin{aligned}
\left\|\left(\widetilde{S} * A^{k-1} B f\right)^{\prime}(t)\right\| & \leq K_{t_{0}} j_{k-\alpha}\left(t_{0}\right) \int_{0}^{t}\left\|A^{k-1} B f(s)\right\| d s \\
& \leq K_{t_{0}} j_{k-\alpha}\left(t_{0}\right)\left\|A^{k-1} B\right\| \int_{0}^{t}\|f(s)\| d s
\end{aligned}
$$

if $k-1<\alpha<k$. Consequently, (2.14) holds, showing that $A+B$ generates a nondegenerate local $\alpha$-times integrated semigroup $T(\cdot)$ on $X$ satisfying (2.7). We deduce from (2.15) and Lemma 2.9 that $T(\cdot)$ is also locally Lipschitz continuous: it suffices to set $Y=\overline{D(A)}, V(\cdot)=S(\cdot), Z(\cdot)=T(\cdot)$ and

$$
W(\cdot)= \begin{cases}S(\cdot) B & \text { if } \alpha=1, \\ \widetilde{S}(\cdot) A^{k-1} B+\sum_{i=0}^{k-2} j_{i+1}(\cdot) A^{i} B & \text { if } 1 \leq k-1<\alpha<k, \\ S(\cdot) A^{k-2} B+\sum_{i=0}^{k-3} j_{i+1}(\cdot) A^{i} B & \text { if } \alpha=k-1 \geq 2,\end{cases}
$$

in Lemma 2.9.

REMARK 2.13. An example in [5, Example 19.11] shows that there exists a nondegenerate $\alpha$-times integrated semigroup on $X$ with a generator $A$ such that $A+B$ does not generate a nondegenerate $\alpha$-times integrated semigroup on $X$ for some bounded linear operator $B$ from $X$ into $D\left(A^{l-1}\right)$.

3. Unbounded perturbation theorems. By slightly modifying the proof of Theorem 2.10, we can establish the next unbounded perturbation theorem concerning local $\alpha$-times integrated semigroups on $X$ which has been obtained by Wang et al. in [25] when $\alpha \in \mathbb{N}$ except for the growth propertis of $T(\cdot)$.

THEOREM 3.1. Let $S(\cdot)$ be a nondegenerate local $\alpha$-times integrated semigroup on $X$ with generator $A$. Assume that $B$ is a bounded linear operator from $[D(A)]$ into $X$ such that $B x \in D\left(A^{l+1}\right)$ for all $x \in D(A)$ and $A+B$ is a closed linear operator from $D(A)$ into $X$. Then $A+B$ generates a nondegenerate local $\alpha$-times integrated semigroup $T(\cdot)$ on $X$ satisfying

$$
T(\cdot) x=S(\cdot) x+D^{\alpha+1} S * B \widetilde{T}(\cdot) x \quad \text { on }\left[0, T_{0}\right)
$$

for all $x \in X$. Here $\widetilde{T}(\cdot)=j_{0} * T(\cdot)$. Moreover, $T(\cdot)$ is also exponentially bounded (resp., norm continuous or exponentially Lipschitz continuous) if $S(\cdot)$ is. 
Proof. We consider only the case $\alpha=k-1 \in \mathbb{N}$, for the case $k-1<\alpha<k$ can be treated similarly. Just as in the proof of Theorem 2.10, for each $0<t_{0}<T_{0}$, we can apply (2.9) and the fact that $B x \in D\left(A^{l+1}\right)$ for all $x \in D(A)$ to establish the following inequalities analogous to (2.10)-(2.13):

$$
\begin{gathered}
\left|S * A^{k-1} B f(t)\right| \leq \sup _{0 \leq r \leq t_{0}}\|S(r)\|\left|A^{k-1} B\right| \int_{0}^{t}|f(s)| d s, \\
\left|j_{i} * A^{i} B f(t)\right| \leq j_{i}\left(t_{0}\right)\left|A^{i} B\right| \int_{0}^{t}|f(s)| d s
\end{gathered}
$$

for all $0 \leq t \leq t_{0}$ and integers $0 \leq i \leq k-2$,

$$
\left|D^{\alpha} S * B f(t)\right| \leq M_{t_{0}} \int_{0}^{t}|f(s)| d s
$$

for all $0 \leq t \leq t_{0}$, and

$$
\left|U^{n} f(t)-U^{n} g(t)\right| \leq M_{t_{0}}^{n} j_{n}\left(t_{0}\right)|f-g|
$$

for all $f, g \in C\left(\left[0, t_{0}\right],[D(A)]\right), 0 \leq t \leq t_{0}$ and $n \in \mathbb{N}$. Here $\left|A^{i} B\right|$ denotes the norm of $A^{i} B$ in $L([D(A)])$ for all integers $0 \leq i \leq k-1,|f-g|=$ $\max _{0 \leq s \leq t_{0}}|f(s)-g(s)|$ and $U: C\left(\left[0, t_{0}\right],[D(A)]\right) \rightarrow C\left(\left[0, t_{0}\right],[D(A)]\right)$ is defined by $U(f)(\cdot)=j_{0} * S(\cdot) x+D^{\alpha}(S * B f)(\cdot)$ on $\left[0, t_{0}\right]$, and

$$
M_{t_{0}}= \begin{cases}\sup _{0 \leq r \leq t_{0}}\|S(r)\|\left|A^{k-1} B\right|+\sum_{i=0}^{k-2} j_{i}\left(t_{0}\right)\left|A^{i} B\right| & \text { if } \alpha=k-1 \in \mathbb{N}, \\ \sup _{0 \leq r \leq t_{0}}\|\widetilde{S}(r)\|\left|A^{k} B\right|+\sum_{i=0}^{k-1} j_{i}\left(t_{0}\right)\left|A^{i} B\right| & \text { if } k-1<\alpha<k .\end{cases}
$$

Combining (3.2)-(3.5), we conclude that for each $x \in X$ there exists a unique function $w_{x}$ in $C\left(\left[0, T_{0}\right),[D(A)]\right)$ such that $w_{x}(\cdot)=j_{0} * S(\cdot) x+$ $D^{\alpha} S * B w_{x}(\cdot)$ on $\left[0, T_{0}\right)$ as in the proof of Theorem 2.10, and then show that $u=j_{0} * w_{x}$ is the unique (strong) solution of $A C P\left(A, j_{\alpha+1}(\cdot) x+j_{0} * B w_{x}, 0\right)$ in $C^{1}\left(\left[0, T_{0}\right), X\right)$, and so $u=j_{0} * w_{x}$ is the unique (strong) solution of $A C P(A+$ $\left.B, j_{\alpha+1}(\cdot) x, 0\right)$ in $C^{1}\left(\left[0, T_{0}\right), X\right)$. Hence $A+B$ generates a nondegenerate local $(\alpha+1)$-times integrated semigroup $\widetilde{T}(\cdot)$ on $X$ satisfying

$$
\widetilde{T}(\cdot) x=j_{0} * S(\cdot) x+D^{\alpha} S * B \widetilde{T}(\cdot) x \quad \text { on }\left[0, T_{0}\right)
$$

for all $x \in X$. From the assumption $B x \in D\left(A^{l+1}\right)$ for all $x \in D(A)$ and (2.9) we see that $\widetilde{T}(\cdot) x$ is continuously differentiable on $\left[0, T_{0}\right)$ for all $x \in X$, and so $T(\cdot)$ defined by $T(t) x=\frac{d}{d t} \widetilde{T}(t) x$ for all $x \in X$ and $0 \leq t<T_{0}$ is a nondegenerate local $\alpha$-times integrated semigroup on $X$ with generator $A+B$ satisfy$\operatorname{ing} T(\cdot) x=S(\cdot) x+D^{\alpha+1} S * B \widetilde{T}(\cdot) x$ on $\left[0, T_{0}\right)$ for all $x \in X$. Clearly, $j_{0} * S(\cdot)$ 
is exponentially Lipschitz continuous if $S(\cdot)$ is exponentially bounded. Applying Lemma $2.8,(2.9)$ and $(3.6)$, we find that $\widetilde{T}(\cdot)$ is exponentially Lipschitz continuous if $S(\cdot)$ is exponentially bounded: just set $Y=[D(A)]$, $V(\cdot)=j_{0} * S(\cdot), Z(\cdot)=\widetilde{T}(\cdot)$ and $W(\cdot)=S(\cdot) A^{k-1} B+\sum_{i=0}^{k-2} j_{i}(\cdot) A^{i} B$ in Lemma 2.8. This implies that $T(\cdot)$ is also exponentially bounded if $S(\cdot)$ is. Next if $S(\cdot)$ is norm continuous (resp., exponentially Lipschitz continuous), then applying Lemma 2.8 again, we infer that $\widetilde{T}(\cdot)$ is also norm continuous (resp., exponentially Lipschitz continuous), and so $A^{i} B \widetilde{T}(\cdot)$ for $0 \leq i \leq k$ are norm continuous (resp., exponentially Lipschitz continuous). Combining this with (2.9), we see that $D^{\alpha+1} S * B \widetilde{T}(\cdot)$ is norm continuous (resp., exponentially Lipschitz continuous), which together with (3.1) implies that $T(\cdot)$ is also norm continuous (resp., exponentially Lipschitz continuous).

By slightly modifying the proof of Theorem 2.12, the next new unbounded perturbation theorem concerning locally Lipschitz continuous local $\alpha$-times integrated semigroups on $X$ is also obtained.

TheOREM 3.2. Let $S(\cdot)$ be a nondegenerate locally Lipschitz continuous local $\alpha$-times integrated semigroup on $X$ with generator $A$ for some $\alpha \geq 1$. Assume that $B$ is a bounded linear operator from $[D(A)]$ into $X$ such that $B x \in D\left(A^{l}\right)$ for all $x \in D(A)$ and $A+B$ is a closed linear operator from $D(A)$ into $X$. Then $A+B$ generates a nondegenerate locally Lipschitz continuous local $\alpha$-times integrated semigroup $T(\cdot)$ on $X$ satisfying (3.1).

Proof. Just as in the proof of Theorem 3.1, we consider only the case $\alpha=k-1 \in \mathbb{N}$, and so for each $0<t_{0}<T_{0}$ and $f \in C\left(\left[0, t_{0}\right],[D(A)]\right)$, we deduce from Remark 2.11 and the fact $\left(S * A^{k-1} B f\right)^{\prime}(\cdot)=A\left(S * A^{k-1} B f\right)(\cdot)+$ $j_{k-2} * A^{k-1} B f$ that (3.4) holds, which implies that $A+B$ generates a nondegenerate local $\alpha$-times integrated semigroup $T(\cdot)$ on $X$ satisfying (3.1). Clearly, $\widetilde{T}(\cdot)$ is locally Lipschitz continuous and $\widetilde{T}(0)=0$ on $X$. It follows that $A^{i} B \widetilde{T}(\cdot)$ is also locally Lipschitz continuous and $A^{i} B \widetilde{T}(0)=0$ on $X$ for all integers $0 \leq i \leq k-1$. Combining this with the local Lipschitz continuity of $S(\cdot)$, we conclude from Remark 2.11 that $\left(S * A^{k-1} B \widetilde{T}\right)^{\prime}(\cdot)$ is locally Lipschitz continuous, which together with $(2.9)$ in which $f$ is replaced by $\widetilde{T}(\cdot)$, and (3.1), implies that $T(\cdot)$ is also locally Lipschitz continuous.

COROllary 3.3. Let $S(\cdot)$ be a nondegenerate local $\alpha$-times integrated semigroup on $X$ with generator $A$. Assume that $B$ is a bounded linear operator from $[D(A)]$ into $X$ such that $B x \in D\left(A^{l+1}\right)$ for all $x \in D(A)$ and $\rho(A+B)$ is nonempty. Then $A+B$ generates a nondegenerate local $\alpha$-times integrated semigroup $T(\cdot)$ on $X$ satisfying (3.1) for all $x \in X$. Moreover, $T(\cdot)$ is also exponentially bounded (resp., norm continuous or exponentially Lipschitz continuous) if $S(\cdot)$ is. 
COROLlary 3.4. Let $S(\cdot)$ be a nondegenerate locally Lipschitz continuous local $\alpha$-times integrated semigroup on $X$ with generator $A$ for some $\alpha \geq 1$. Assume that $B$ is a bounded linear operator from $[D(A)]$ into $X$ such that $B x \in D\left(A^{l}\right)$ for all $x \in D(A)$ and $\rho(A+B)$ is nonempty. Then $A+B$ generates a nondegenerate locally Lipschitz continuous local $\alpha$-times integrated semigroup $T(\cdot)$ on $X$ satisfying (3.1).

When the assumption that $A+B$ is a closed linear operator from $D(A)$ into $X$ is replaced by assuming that $A B=B A$ on $D\left(A^{2}\right)$, we can obtain the next unbounded perturbation result which has been obtained by Wang et al. in [25] when $\alpha \in \mathbb{N}$ except for the growth properties of $T(\cdot)$.

COROLlary 3.5. Let $S(\cdot)$ be a nondegenerate local $\alpha$-times integrated semigroup on $X$ with generator $A$. Assume that $B$ is a bounded linear operator from $[D(A)]$ into $X$ such that $B x \in D\left(A^{l+1}\right)$ for all $x \in D(A)$ and $A B=B A$ on $D\left(A^{2}\right)$. Then $A+B$ generates a nondegenerate local $\alpha$-times integrated semigroup $T(\cdot)$ on $X$ satisfying

$$
T(\cdot) x=S(\cdot) x+D^{\alpha} S *(\lambda-A) B(\lambda-A)^{-1} T(\cdot) x \quad \text { on }\left[0, T_{0}\right)
$$

for all $x \in X$. Here $\lambda \in \rho(A)$. Moreover, $T(\cdot)$ is also exponentially bounded (resp., norm continuous or exponentially Lipschitz continuous) if $S(\cdot)$ is.

Proof. Just as in the proof of [25, Theorem 3.1], we can show that $A+B$ is a closed linear operator from $D(A)$ into $X$, or equivalently, $\lambda-(A+B)$ is. Here $\lambda \in \rho(A)$ is fixed. By slightly modifying the proof of Theorem 2.10, we also deduce that for each $x \in X$ there exists a unique function $w_{x}$ in $C\left(\left[0, T_{0}\right), X\right)$ such that $w_{x}=S(\cdot) x+D^{\alpha} S *(\lambda-A) B(\lambda-A)^{-1} w_{x}$, and so $j_{0} * w_{x}$ is the unique solution of

$$
\begin{aligned}
A C P\left(A, j_{\alpha} x+j_{0} *(\right. & \left.\lambda-A) B(\lambda-A)^{-1} w_{x}, 0\right) \\
& =A C P\left(A, j_{\alpha} x+(\lambda-A) B(\lambda-A)^{-1} j_{0} * w_{x}, 0\right) \\
& =A C P\left(A, j_{\alpha} x+(\lambda-A) B(\lambda-A)^{-1} j_{0} * w_{x}, 0\right) \\
& =A C P\left(A, j_{\alpha} x+B j_{0} * w_{x}, 0\right)
\end{aligned}
$$

in $C^{1}\left(\left[0, T_{0}\right), X\right)$. Hence $u=j_{0} * w_{x}$ is the unique function in $C^{1}\left(\left[0, T_{0}\right), X\right)$ such that $u^{\prime}=A u+j_{\alpha} x+B u=(A+B) u+j_{\alpha} x$ on $\left[0, T_{0}\right)$ and $u(0)=0$. Applying Theorem 2.4 again, we find that $A+B$ generates a nondegenerate local $\alpha$-times integrated semigroup on $X$ satisfying (3.7) which is defined by $T(\cdot) x=w_{x}(\cdot)$ for all $x \in X$. Moreover, $T(\cdot)$ is also exponentially bounded (resp., norm continuous or exponentially Lipschitz continuous) if $S(\cdot)$ is.

By slightly modifying the proof of Theorem 2.12, the next unbounded perturbation result concerning locally Lipschitz continuous local $\alpha$-times integrated semigroups on $X$ is also obtained. 
COROllary 3.6. Let $S(\cdot)$ be a nondegenerate locally Lipschitz continuous local $\alpha$-times integrated semigroup on $X$ with generator $A$ for some $\alpha \geq 1$. Assume that $B$ is a bounded linear operator from $[D(A)]$ into $X$ such that $B x \in D\left(A^{l}\right)$ for all $x \in D(A)$ and $A B=B A$ on $D\left(A^{2}\right)$. Then $A+B$ generates a nondegenerate locally Lipschitz continuous local $\alpha$-times integrated semigroup $T(\cdot)$ on $X$ satisfying (3.7).

We end this paper with a simple illustrative example. Let $X=L^{\infty}(\mathbb{R})$, and $A: D(A) \subset X \rightarrow X$ be defined by $D(A)=W^{1, \infty}(\mathbb{R})$ and $A f=$ $-f^{\prime}$ for all $f \in D(A)$. Then $A$ generates a locally Lipschitz continuous local 1-times integrated semigroup $S(\cdot)\left(=\left\{S(t) \mid 0 \leq t<T_{0}\right\}\right)$ on $X$ and $\overline{D(A)}=C_{0}(\mathbb{R})$ (see [1, Example 3.3.10]). Here $0<T_{0} \leq \infty$ is fixed. Applying Theorem 2.12, we find that $A+B$ generates a locally Lipschitz continuous local 1-times integrated semigroup $T(\cdot)$ on $L^{\infty}(\mathbb{R})$ satisfying $(2.7)$ when $B$ is a bounded linear operator from $C_{0}(\mathbb{R})$ into $L^{\infty}(\mathbb{R})$ defined by $B(f)(t)=\int_{-\infty}^{\infty} f(t-s) d \mu(s)$ for all $f \in C_{0}(\mathbb{R})$ and $t \in \mathbb{R}$. Here $\mu$ is a fixed finite regular Borel measure on $\mathbb{R}$.

Acknowledgements. The author is grateful to the referee for his or her corrections and valuable suggestions.

This research was partially supported by the National Science Council of Taiwan.

\section{References}

[1] W. Arendt, C. J. K. Batty, M. Hieber and F. Neubrander, Vector-Valued Laplace Transforms and Cauchy Problems, Birkhäuser, Basel, 2001.

[2] W. Arendt and H. Kellermann, Integrated solutions of Volterra integrodifferential equations and applications, in: Pitman Res. Notes Math. 190, Longman, Harlow, 1989, 21-51.

[3] W. Arendt, O. E. I-Mennaoui and V. Keyantuo, Local integrated semigroups: evolution with jumps of regularity, J. Math. Anal. Appl. 186 (1994), 572-595.

[4] W. Arendt, F. Neubrander and U. Schlotterbeck, Interpolation of semigroups and integrated semigroups, Semigroup Forum 45 (1992), 26-37.

[5] R. deLaubenfels, Existence Families, Functional Calculi and Evolution Equations, Lecture Notes in Math. 1570, Springer, Berlin, 1994.

[6] —, Integrated semigroups, C-semigroups and the abstract Cauchy problem, Semigroup Forum 41 (1990), 83-95.

[7] M. Hieber, Integrated semigroups and differential operators on $L^{p}$ spaces, Math. Ann. 291 (1991), 1-16.

[8] -, Laplace transforms and $\alpha$-times integrated semigroups, Forum Math. 3 (1991), 595-612.

[9] H. Kellermann and M. Hieber, Integrated semigroups, J. Funct. Anal. 84 (1989), 160-180.

[10] C.-C. Kuo, On existence and approximation of solutions of abstract Cauchy problem, Taiwanese J. Math. 13 (2009), 137-155. 
[11] C.-C. Kuo, On perturbation of $\alpha$-times integrated $C$-semigroups, ibid., to appear.

[12] C.-C. Kuo and S.-Y. Shaw, On $\alpha$-times integrated $C$-semigroups and the abstract Cauchy problem, Studia Math. 142 (2000), 201-217.

[13] - - - Strong and weak solutions of abstract Cauchy problems, J. Concrete Appl. Math. 2 (2004), 191-212.

[14] M. Li and Q. Zheng, $\alpha$-Times integrated semigroups: local and global, Studia Math. 154 (2003), 243-252.

[15] Y.-C. Li and S.-Y. Shaw, Perturbation of non-exponentially-bounded $\alpha$-times integrated C-semigroups, J. Math. Soc. Japan 55 (2003), 1115-1136.

[16] - - -, On local $\alpha$-times integrated C-semigroups, Abstract Appl. Anal. 2007, art. ID 34890, 18 pp.

[17] M. Mijatović and S. Pilipović, $\alpha$-Times integrated semigroups $\left(\alpha \in \mathbb{R}^{+}\right)$, J. Math. Anal. Appl. 210 (1997), 790-803.

[18] I. Miyadera, M. Okubo and N. Tanaka, On integrated semigroups which are not exponentially bounded, Proc. Japan Acad. 69 (1993), 199-204.

[19] F. Neubrander, Integrated semigroups and their applications to the abstract Cauchy problem, Pacific J. Math. 135 (1988), 111-155.

[20] - Integrated semigroups and their applications to complete second order Cauchy problems, Semigroup Forum 38 (1989), 233-251.

[21] S. Nicaise, The Hille-Yosida and Trotter-Kato theorems for integrated semigroups, J. Math. Anal. Appl. 180 (1993), 303-316.

[22] H. Oka, Linear Volterra equations and integrated solution families, Semigroup Forum 53 (1996), 278-297.

[23] S.-Y. Shaw and C.-C. Kuo, Generation of local C-semigroups and solvability of the abstract Cauchy problems, Taiwanese J. Math. 9 (2005), 291-311.

[24] N. Tanaka and N. Okazawa, Local C-semigroups and local integrated semigroups, Proc. London Math. Soc. 6 (1990), 63-90.

[25] S. W. Wang, M. Y. Wang and Y. Shen, Perturbation theorems for local integrated semigroups and their applications, Studia Math. 170 (2005), 121-146.

[26] T. J. Xiao and J. Liang, The Cauchy Problem for Higher-Order Abstract Differential Equations, Lecture Notes in Math. 1701, Springer, 1998.

[27] Q. Zheng, Perturbations and approximations of integrated semigroups, Acta Math. Sci. 9 (1993), 252-260.

Chung-Cheng Kuo

Department of Mathematics

Fu Jen University

Taipei, Taiwan 24205

E-mail: cckuo@math.fju.edu.tw

Received March 2, 2009

Revised version August 31, 2009

(6561) 